\title{
Role of Knowledge Engineering in the Development of a Hybrid Knowledge Based Medical Information System for Atrial Fibrillation
}

\author{
Aankita Kaur ${ }^{1}$, Mansaf Alam² \\ ${ }^{1}$ Department of Mathematics, Jamia Millia Islamia University, New Delhi, India; ${ }^{2}$ Department of Computer Science, Jamia Millia \\ Islamia University, New Delhi, India. \\ Email: aankitakaur@gmail.com,mansaf_alam2002@yahoo.com
}

Received October $9^{\text {th }}, 2012$; revised November $13^{\text {th }}$, 2012; accepted December $15^{\text {th }}, 2012$

\begin{abstract}
In this paper, we are describing role of knowledge engineering in the development of a hybrid knowledge based medical information system. Knowledge engineering plays important role in development of various technologies such as: expert systems, neural network, artificial intelligence, hybrid intelligent systems, data mining, decision support systems, and knowledge based systems etc. The hybrid medical information system mainly consists of medical information system and medical knowledge base systems. These poly techniques of knowledge engineering when integrated with hybrid techniques of intelligent systems for designing, implementing knowledge bases to deal with medical informational data of Atrial Fibrillation. Atrial Fibrillation is the most common heart rhythm disorder which increases the risk of mortality and morbidity.
\end{abstract}

Keywords: Artificial Intelligence (AI); Decision Making; Knowledge Engineering; Knowledge Base; Mortality; Morbidity; Knowledge Management (KM); Experience Management (EM); Knowledge Base System (KBS); Knowledge Management System (KMS); Atrial Fibrillation (AFib); Knowledge Discovery Techniques (KDT); Medical Information System; Medical Information Based Decision Making; Hybrid Intelligent Medical Information System (HIMIS); Hybrid Knowledge Based System

\section{Introduction}

Knowledge Engineering plays a vital role in the development of various technologies. These technologies are expert systems, neural network, artificial intelligence, intelligent systems, data mining, decision support systems, knowledge based systems etc. These technologies are used in hybrid fashion that is combining of them with their strengths and weaknesses to develop Hybrid Knowledge based medical information system.

The development of Hybrid knowledge based medical information system needs the useful medical data. The useful medical data must be chosen carefully, studied and interpreted in a proper manner to develop knowledge based medical information system. The aim of this study is to propose a new system which is based on combining hybrid techniques of intelligent systems for designing, implementing knowledge base to deal with medical informational data of Atrial Fibrillation.

The knowledge base (KB) consists of collection of related knowledge to solve any problem. Knowledge based systems (KBS) are an important area in artificial intel- ligence (AI) in which knowledge base (KB) and inference engine are the main components [1]. Knowledge management system (KMS) facilitates the flow of knowledge from one source(s) which knows another source(s) in the updated system. The knowledge data collected needs proper management.

Knowledge management (KM) is an approach of managing and sharing information stored in a database which supports the decision making process. The acquired is used in business management decision making. Integrating Knowledge management and decision making system can be done faster and more accurately with the help of knowledge discovery techniques of data mining.

The knowledge without experience is less meaningful. If combined together can do marvels. The experiences can be any previous or super specialized knowledge. For an example: If you visited any hospital for a regular check up, and a practitioner examines you and tells that you are fine. This is a typical experience. Only a previous knowledge with a problem and a successful solution to the problem is experience [1]. Sometimes ex- 
perience (practice of knowledge) and medical check tests are combination of knowledge and experience for an expert KMS.

Experience management (EM) is a tool which focuses on experience processing and corresponding to management [2]. It stores information directly captured from fresh experiences also. These fresh specific experiences containing actionable information are added to knowledge which is further used for processing and management of new experiences. Hence Experience and Knowledge Management both are interrelated and important in modeling of an intelligent system contributing to artificial intelligence. Knowledge management (KM) plays an important role in business management, Information technology, artificial intelligence (AI) also [3,4].

Artificial Intelligence gives solutions for complex problems based on human expertise, logic and reasoning which are stored in a computer. AI techniques as neural network and expert systems are capable of designing intelligent machines that can perform even better than humans for Atrial Fibrillation.

Atrial Fibrillation is the most common heart rhythm disorder. It also increases the risk of stroke, heart diseases, mortality, morbidity and premature deaths [5,7]. In this type of disorder (arrthymia); the heart beats either too fast or too slow with irregular rhythm [8]. The risk of Atrial Fibrillation increases with various parameters such as old age, diabetes, hypertension, Obstructive sleep apnea (OSA), Myocardial Infraction, Pulmonary diseases, etc.

In modern medicine a large amount of medical data is witnessed from a practitioner, the nursing staff, the patient care, the expert's opinions and many other sources. Since lots of data is to be managed for medical purposes, datasets are large in quantity and heterogeneous in nature. Theses medical data records are stored and updated electronically by medical staff members in the form of database. Therefore, some scheme of decision and planning is followed to retrieve useful information from all of these stored medical records. This provides useful information and interesting patterns for future use in medical sciences. It constructs a record keeping system of medical information for prediction and management of serious diseases.

The rest of the paper is organized as follows: Section 2 describes the role of decision making process and methods of reasoning in medical information system, Section 3 highlights the role of expert systems in construction of knowledge base systems, Section 4 introduces different knowledge based systems for Atrial Fibrillation disease. Sections 5 and 6 look after the Hybrid medical information system for Atrial Fibrillation with knowledge base systems. The final section concludes the paper.

\section{Medical Information System Based on Decision Making Processes}

Decision Making Processes for solving a medical problem are done by providing a set of tools. These tools assist the medical decision making situation with improved the outcomes. The medical data are heterogeneous in nature. Since medical decision making basically depends on medical data diagnostics, information and datasets. These datasets store various information's for example: patient's history, symptoms, laboratory reports, causes and effects, line of treatments, practitioner's diagnosis, nursing staff etc. To maintain database for future use there is a need to keep Medical Information System. This Medical Information System contains various wings such as: Accounts, Billing, Pharmacy, Patient's care, Emergency records keeping, In/Out patient records and various other information kept in the form of Remarks.

In modeling of a problem based on decision making in medical information system we use knowledge engineering methods. There are different methods of reasoning extractions to support medical decision support system which are as follows $[9,10]$.

Case Based Reasoning: In case based reasoning [11], records of past case studies are used. It is done by backward chaining for solving new problems which are featured similar to old ones. These collections of cases which are stored in a database are called case base, way of knowledge representation.

Rule Based Reasoning: In rule based reasoning, the classes are used for solving problems with thumb rules. It is solved by forward chaining. These rules are made with combined experiences which are usually in the form of IF $<$ a problem $>$ THEN < set of conditions $>$, here AND, OR, ELSE can also be added to increase the steps of procedures easing the problem solving rules.

Probabilistic Reasoning: In probabilistic reasoning, the probability of uncertainty guides the system by using bays theorem of conditional probability to automatically take the decision. The most probable classes hint in finding the likelihood of the outcome to the solution of the problem [12]. The results are further statistically and mathematically verified.

Experience Based Reasoning: In experience based reasoning [13], the specific actionable experiences are added to the knowledge retrieval for solving different type of cases. It is also by adding self decision, intuition, feeling, and wisdom, and common sense. The different experiences are further processed and managed.

\section{Expert Systems with Artificial Intelligence in Development of Knowledge Based Systems}

Expert Systems use human decision making processes to 
build a knowledge based components in computer. A knowledge engineer must utilize proper skills to extract knowledge (domain specific and general purpose) from human experts which can be placed in knowledge based systems. These expert systems capture knowledge from various experts such as: retired staff, different methodologies, expert's experiences, heuristic (trials and errors) knowledge, intellect built, intuition gained and wisdom powers of experts which provide intelligent advice/decisions.

The main components of an expert system are: a knowledge base where the knowledge is stored for various representation schemes and decision making processes are build, inference engine which is operated on the knowledge base to provide reasons and get conclusions, explanatory interface which interacts with human and providing explanation on how expert system gives final conclusions, knowledge acquisition which acquire sufficient knowledge from human experts to build new knowledge base.

Medical Knowledge is extracted by collecting views from various sources such as: the domain expert's knowledge, the medical literature, interviewing patients/relatives, the practitioners, the nursing staff, the specialists and the multiple expert opinions on the modeling problem. These are the steps how an expert system works from knowledge retrieval by experts to finally integrating it into knowledge base systems of various Atrial Fibrillation problems.

\subsection{Knowledge Retrieval from Expert}

An expert's plays the role of a specialist's in their particular domain of knowledge. Expert's posses detailed and in depth knowledge about their subject of interest. These interest areas can be an item, event, a task performed, meta-knowledge (knowledge about knowledge) etc. Expert knowledge is retrieved in the following steps: firstly there is a case analysis of the experiences gathered by experts, then other critical incidents are viewed, discussions are carried forward with the users. Designing of a prototype model for reasoning and planning of a particular problem is done and then lastly performances are reviewed by validation and verification of the knowledge obtained by the experts.

\subsection{Knowledge Acquisition and Reasoning}

Knowledge acquisition is the process in which knowledge engineers acquire knowledge from human experts, group of experts, documentation, manuals, which helps in building complete, accurate and well organized knowledge based systems. Knowledge is acquired in the form of interviews (unstructured, structured, event recall, vast thinking) that can introduce some important topics, observations, filling questionnaires from patients/relatives, Expert Consultants/Practitioners etc. The role of recalling focuses on completion of knowledge obtained by cross checking expert's knowledge obtained in the form of reasoning how and why. Group of experts giving their respective opinion can create internal conflicts. They might use different reasoning steps. Therefore, there must be a judge who is specialized in that problem domain with more experiences gathered to conclude the steps to be followed in a specific decision making process.

\subsection{Knowledge Representation and Reasoning}

The knowledge available in the previous steps must be represented in an efficient manner. Knowledge engineer must have clear understanding of the representation scheme so that problem can be easily handled. Knowledge can be declarative or procedural. Declarative knowledge is a sentence depicting facts while procedural knowledge is a group of sentences, which can be carried in a series of steps by building relationships. Knowledge must be encoded either as a part of expert systems or should be easily available to them. An Inference engine detects these knowledge representation schemes which are in the form of the following: rules, semantic nets, frames and ontology. At times, there could be multiple knowledge representation schemes that describe the given problem.

Rule based reasoning as described in previous section depicts how an expert gives explanation about the conclusion with the help of rules. But it cannot describe why these rules apply on the problem. This could be because of insufficient knowledge about a particular subject domain in the knowledge base system. Semantic net is a graphical representation of nodes (objects) labeled and followed by links (relationships) to describe knowledge in a pictorials manner. Knowledge engineers understand these networks easily as they are presented in the hierarchical manner of using expert's knowledge. Frames are also hierarchal in nature. They also give a simplified method of storing knowledge in form of a table. The knowledge used in one frame can inherit properties used in another frame. Ontology refers to representations of specific standard symbols which are used in knowledge, expert systems and knowledge base systems for the particular purpose.

\subsection{Construction of Knowledge Base from Intelligent Systems}

Knowledge is most important for building an intelligent system. Artificial Intelligence focuses on development of various models, designing of knowledge representation schemes which can be useful in solving complex applications. For example: Natural language processing uses 
user defined language in order to easily interact with machine for solving a problem. Knowledge base must contain enough knowledge that can support user queries and should be easy to use. Knowledge base must contain the following documentation: User documentation which provides guidance to different users on how to use knowledge base systems. Technical documentation must also be provided which should have depth of technical knowledge in it.

\section{Modeling of Knowledge Based Systems: Atrial Fibrillation}

The collection of knowledge related to Atrial Fibrillation (heart rhythm disorder) problem can be used in an Artificial Intelligence system. It is organized and managed in different knowledge based systems. To solve an Atrial Fibrillation problem, single or multiple knowledge base systems can be used to extract various knowledge representation schemes that define the problem. These expertise systems grow as more representation schemes are added. These systems are easily processed, documented, reused and accessed while dealing with a different problem.

Our model is created and evaluated using the datasets collected from cardiology department of a hospital on Atrial Fibrillation. The dataset consists of 258 patients records and 255 classified attributes (categorical, binary, numeric etc.). The knowledge based systems for storing different representation schemes which facilitates the problem of solving Arial Fibrillation are as follows [14].

\subsection{KBS 1: Types of Atrial Fibrillation}

Atrial Fibrillation can mainly be distributed in three categories on the basis of how long heart beats in an irregular rhythm. This can be noted in an EGC. If an episode is more than 30 seconds long (only those are taken care by a Practitioner's records). Episodes that are terminated by itself within 7 days are categorized into paroxysmal, longer than 7 days or terminated by treatment falls into persistent types and episodes which last longer than one year are termed as permanent type of Atrial Fibrillation. In ECG the absence of $\mathrm{P}$ wave also depicts the chances of Atrial Fibrillation whereas Atrial flutter can also be noted in the similar manner. There is a difference between the two of them that is Atrial flutter is more organized than Atrial Fibrillation.

\subsection{KBS 2: Attributes That Triggers Atrial Fibrillation}

Atrial Fibrillation can be triggered by many factors. There are some factors which are more risky when other factors also exist in the life style. For example: If a male person is already 60 years of age have adopted a life style of excessive usage of tobacco, has a family history of coronary heart disease and is now diagnosed with diabetes also. There is another male who is of the same age but has adopted a healthy life style; the former has more chances of getting effected by Atrial Fibrillation than the later one.

There are other medical symptoms also that increase the chances of affecting Atrial Fibrillation that are as follows: Valvular heart disease, Stroke, Ischemic heart disease, chest discomfort, blood pressure, irregular pulse rate, dizziness, having undergoing heart surgery, Palpitations etc.

\section{A Hybrid Medical Information System for Atrial Fibrillation}

A Hybrid Medical Information System includes acquiring information from system, documentation and other technical information to modify or develop medical information system [15,16]. It requires several knowledge representation approaches to construct frames and rules.

An intelligent integration of diagnosis and treatment of Atrial Fibrillation patients consulting a specialist (cardiologist) is an example of Hybrid Intelligent Medical Information System (HIMIS) as described in Figure 1 [16$18]$.

The main components of intelligent integrated Atrial Fibrillation patient systems medical information system are:

- A Patient Record system;

- A Patient diagnosis and treatment (DT) system;

- An Intelligent Consultation system.

The patient record system maintains patient medical records, gender, age, clinical reports and results, past history, allergies, diabetes, blood pressure, pulse rate, medication prescribed, practitioner's records and patient care.

The patient diagnosis and treatment system is a knowledge base used by practitioners in solving uncommon

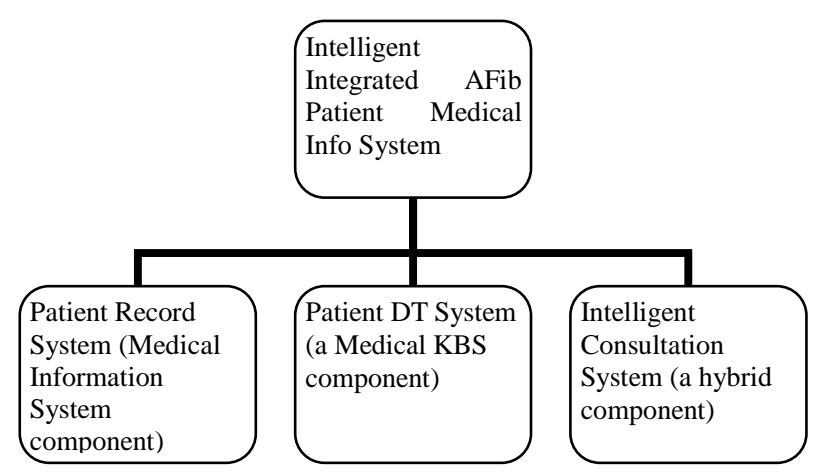

Figure 1. Hybrid intelligent medical information system for Atrial Fibrillation patient. 
and difficult cases. As systems are integrated so there is an easy access for records to be shared and updated in the patient record system

The intelligent consultation system is highly coupled. A consultation system with added diagnosis and treatment (DT) knowledge offers consultation on the priority of emergency, urgent, ICU, OPD, private, non urgent, special quota.

A hybrid intelligent medical information system combines medical information system and medical KBSs. There are various other component wings for storing useful medical information in the form of medical datasets the helps in construction of HIMIS are as follows: Pharmacy, Accounts department, Billing, In/Out patient registration history, Consultant's detailed records, medical insurance company and others which collect some extra valuable special features.

\section{Hybrid Knowledge Based Medical Information System for Atrial Fibrillation}

The role of using hybrid systems is to increase the strengths of a system and decreasing the weaknesses present in one system by combining another system judiciously in an efficient manner. Hybrid Intelligence has also been an important in medical decision making.

The concept of coupling an original system with knowledge based system (KBS) which helps in achieving a specific task. There are different knowledge based systems such as expert systems, neural networks, probabilistic reasoning systems, case base system etc.

Neural networks have ability to learn from examples so they can be trained easily, work fast as they are connected in parallel for processing but cannot explain how steps are processed. Expert systems have clarity of representation and processing steps though they are slow. Many research works have been done by combining hybrid system connectionist subsystem and an expert system [19-21].

Here we couple neural network as shown in Figures 2 and $\mathbf{3}$ and an expert system which is already described. The output of neural network is taken as input in an expert system. Consider a scenario where a patient suffers from Atrial Fibrillation. The input layer consists of types of Atrial Fibrillation: Paroxysmal, Persistent, Permanent with hidden layer marked as 1, 2 and 3. Also in another system the attributes such as stroke, old age, diabetes, obstructive sleep apnea, coronary heart disease, hypertension, excessive smoking and drinking habits, stress and depression, heavy exercise that triggers the condition of Atrial Fibrillation. A Patient with hidden layers marked with different diagnosis and treatment which gives mortality or morality as final outcomes. Here we have combined two neural networks one having types of Atrial

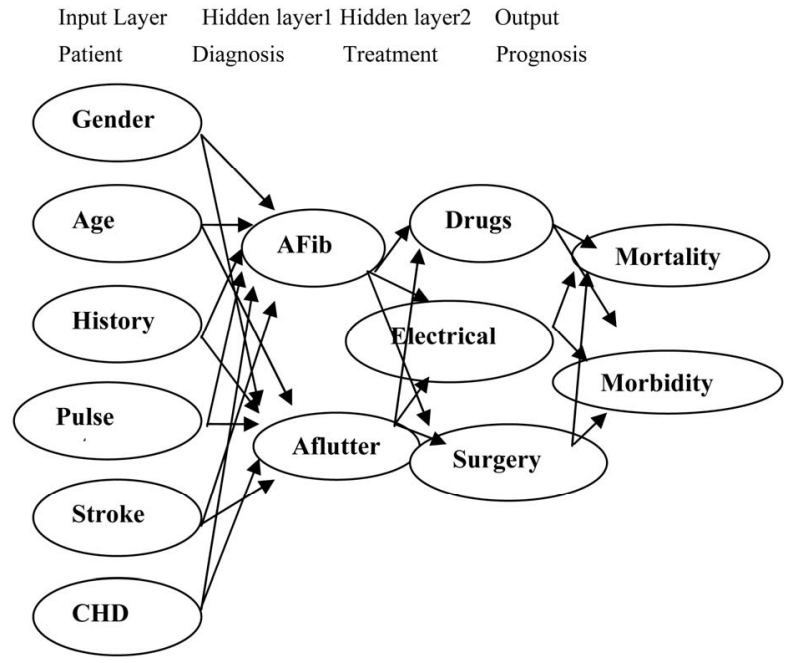

Figure 2. Neural network for those attributes which triggers the cause of mortality and morbidity.

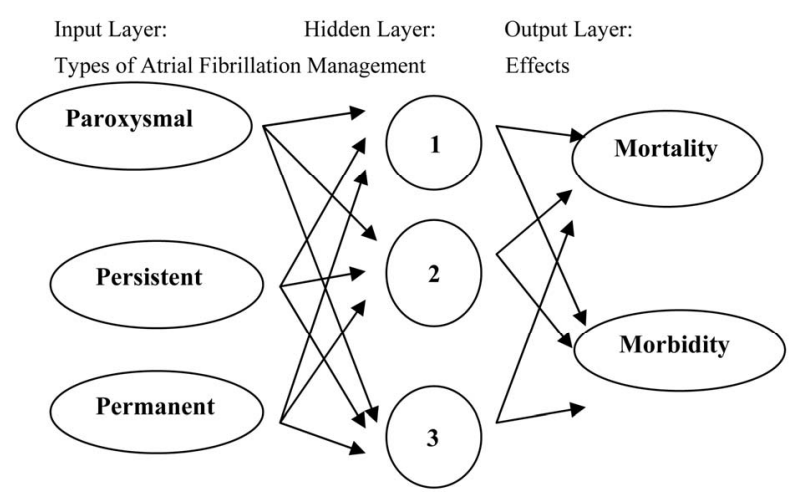

Figure 3. Neural network for types of atrial fibrillation reason for mortality (death) or morality (causing within a population).

Fibrillation and other the main attributes that triggers Atrial Fibrillation. Combing these two systems will give us results for mortality and morality. The reasoning part is taken care by expert system which is hybridized later by showing the appropriate rule such as:

IF Atrial Fibrillation is Permanent, Stroke and Hypertension THEN Mortality.

\section{Conclusion}

We have presented a new system which is based on combining hybrid techniques of intelligent systems for designing, implementing knowledge bases to deal with medical informational data of Atrial Fibrillation. Atrial Fibrillation is the most common heart rhythm disorder which increases the risk of mortality and morbidity. The hybrid model constructed uses intelligent medical information system with decision making support system of Atrial Fibrillation, different knowledge based systems of Atrial Fibrillation prepared in an expert system with ar- 
tificial intelligence techniques such as neural networks and other technologies used by Knowledge Engineers. The future extension of this paper includes developing some new trends, dependencies of the medical information data for Atrial Fibrillation [22,23]. This will deal with assumptions on performance of various other data mining techniques integrated with knowledge driven approach applied in mathematical formulation. It will be done by applying the best technique to handle certain problem under consideration that will further help in Management Information System.

\section{REFERENCES}

[1] Z. Sun and G. Finnie, "Intelligent Techniques in E-Commerce: A Case-Based Reasoning Perspective,” SpringerVerlag, Heidelberg, 2004.

[2] R. Bergmann, "Experience Management: Foundations, Development Methodology and Internet-Based Applications,” Springer, Berlin, 2002.

[3] Z. Sun, “A Waterfall Model for Knowledge Management and Experience Management,” Proceedings of International Conference on Hybrid Intelligent Systems, Kitakyushu, 6-8 December 2004, pp. 472-475.

[4] H. Hasan and M. Handzic, "Australian Studies in Knowledge Management,” University of Wollongong Press, Wollongong, 2003.

[5] E. J. Benjamin, P. A. Wolf, R. B. D. Agostino, et al., "Impact of Atrial Fibrillation on the Risk of Death: The Framingham Heart Study," Circulation, Vol. 98, No. 10, 1998, pp. 946-952.

[6] K. Lakshminarayan, C. A. Solid, A. J. Colins, et al., “Atrial Fibrillation and Stroke in the General Medicare Population-A 10-Year Perspective (1992 to 2002)," Stroke, Vol. 37, No. 8, 2006, pp. 1969-1974. doi:10.1161/01.STR.0000230607.07928.17

[7] P. A. Wolf, R. D. Abbott and W. B. Kanne, "Atrial Fibrillation as an Independent Risk Factor for Stroke: The Framingham Heart Study,” Stroke, Vol. 22, 1991, pp. 983988. doi:10.1161/01.STR.22.8.983

[8] T. Thom, N. Haase, W. Rosamond, et al., "Heart Disease and Stroke Statistics-A Report from American Heart Association Statistics Committee and Stroke Statistics Subcommittee," Circulation, Vol. 113, 2006, p. e85.

[9] K. E. Burn-Thornton, "Making the Most of Data in Order to Provide Accurate Clinical Decision Support Systems for the Use in the Determination of Heart Disease," Proceeding of 3rd International Conference on Data Mining, Bologna, 25-27 September 2002, pp. 839-848.

[10] I. Watson, “Applying Case-Based Reasoning: Techniques for Enterprise Systems,” Morgan Kaufmann Publishers, San Francisco, 1997.

[11] J. Surma and K. Vanhoff, "Integrating Rules and Cases for ICCBR,” Lecture Notes in Artificial Intelligence 1010, Sesimbra, 1995.

[12] S. Montani, R. Bellazzi, L. Portinale, S. Fiocchi and M. A.
Stefanelli, "Case-Based Retrieval System for Diabetic Patients Therapy,” Proceedings of IDAMAP 98 Working Notes, Workshop ECAI 98, Brighton, 1998, pp. 64-70.

[13] Z. Sun and G. Finnie, "Experience Based Reasoning for Recognizing Fraud and Deception,” Proceedings of International Conference on Hybrid Intelligent Systems (HIIS 2004), Kitakyushu, 6-8 December 2004, pp. 80-85.

[14] A. Kaur, "Hybrid Approach: Predictive Data Mining Model for Atrial Fibrillatiom," International Conference on World Congress on Information and Communication Technologies, Mumbai, 11-14 December 2012, pp. 126-131. doi:10.1109/WICT.2011.6141230s

[15] S. L. Kendal, K. Ashton and X. Chen, “A Brief Overview of HyM: A Methodology for the Development of Hybrid Intelligent Information Systems,” Proceedings of the 15th International Conference on Software Engineering and Knowledge Engineering, San Francisco, 1-3 July 2003, pp. 322-326.

[16] U. Rashad, P. Arullendran, M. Hawthorne and S. Kendal, "A Hybrid Medical Information System for Diagnosis of Dizziness,” Proceedings of the 4th International Conference on Neural Networks and Expert Systems in Medicine and Healthcare, Milos Island, 20-22 June 2001.

[17] S. L. Kendal, K. Ashton and X. Chen, "A Brief Review of HyM: A Methodology for the Development of Hybrid Intelligent Information Systems," Proceedings of the 15th International Conference on Software Engineering and Knowledge Engineering, San Francisco, 1-3 July 2003, ISBN: 1-891706-12-8.

[18] S. L. Kendal, X. Chen and A. Masters, "HyM: A Methodology for Development of Integrated Hybrid Intelligent Information Systems,” Proceedings of Fusion 2000-3rd International Conference on Information Fusion, Paris, 10-13 July 2000, pp. WEC4/17-WEC4/22.

[19] G. G. Towell, J. W. Shavlik and M. O. Noordwier, "Refinement of Approximate Domain Theories by Knowledge Based-Artificial Neural Network," Proceedings of the 8th National Conference on Artificial Intelligence, Boston, July 1990, pp. 861-866.

[20] A. B. Tickle, R. Andrews, M. Goela and J. Diederich, "The Truth Will Come to Light: Directions and Challenges in Extracting the Knowledge Embedded within Trained Artificial Neural Networks,” IEEE Transactions on Neural Networks, Vol. 9, No. 6, 1998, pp. 1057-1068.

[21] L. M. Fu, "Knowledge Based Connectionism for Revising Domain Theories," IEEE Transactions on Systems, Man, and Cybernetics, Vol. 23, No. 1, 1993, pp. 173-182. doi:10.1109/21.214775

[22] M. G. Tsipouras, D. I. Fotiadis and D. Sideris, “An Arrhythmia Classification System Based on the RR-Interval Signal,” Artificial Intelligence in Medicine, Vol. 33, No. 3, 2005, pp. 237-250. doi:10.1016/j.artmed.2004.03.007

[23] B. M. Asl, S. K. Setarehdan and M. Mohebbi, "Support Vector Machine-Based Arrhythmia Classification Using Reduced Features of Heart Rate Variability Signal,” Artificial Intelligence in Medicine, Vol. 44, No. 1, 2008, pp. 51-64. doi:10.1016/j.artmed.2008.04.007 